\title{
落叶松人工林细根动态与土壤资源有效性关系研究
}

\author{
程云环 ${ }^{1}$ 韩有志 ${ }^{1,2}$ 王庆成 ${ }^{1}$ 王政权 ${ }^{1 *}$ \\ （1 东北林业大学林学院, 哈尔滨 150040）（2 山西农业大学林学院,太谷 030801）
}

摘 要 树木细根在森林生态系统 $\mathrm{C}$ 和养分循环中具有重要的作用。由于温带土壤资源有效性具有明显的季节 变化, 导致细根生物量、根长密度 (Root length density, RLD) 和比根长 (Specific root length, SRL) 的季节性变化。以 17 年生落叶松 (Larix gmelini) 人工林为研究对象, 采用根钻法从 5 月到 10 月连续取样, 研究了不同土层细根 (直径 $\leqslant 2$ $\mathrm{mm}$ ) 生物量、 $R L D$ 和 $S R L$ 的季节动态, 以及这些根系指标动态与土壤水分、温度和 $\mathrm{N}$ 有效性的关系。结果表明: 1 ) 落叶松细根年平均生物量 (活根 + 死根) 为 $189.1 \mathrm{~g} \cdot \mathrm{m}^{-2} \cdot \mathrm{a}^{-1}$, 其中 $50 \%$ 分布在表层 $(0 \sim 10 \mathrm{~cm}), 33 \%$ 分布在亚表层 (11 20 cm), 17\% 分布在底层 $(21 \sim 30 \mathrm{~cm}$ )。活根和死根生物量在 5 7 月以及 9 月较高, 8 月和 10 月较低。从春季 (5 月)到秋季 (10月), 随着活细根生物量的减少, 死细根生物量增加; 2 ) 土壤表层 (0 10 cm) 具有较高的 $R L D$ 和 $S R L$, 而底层 (21 30 cm) 最低。春季( 5 月)总 $R L D$ 和 $S R L$ 最高, 分别为 $10621.45 \mathrm{~m} \cdot \mathrm{m}^{-3}$ 和 $14.83 \mathrm{~m}^{\bullet} \mathrm{g}^{-1}$, 到秋季( 9 月)树木生长结束后达到最低值, 分别为 $2198.20 \mathrm{~m} \cdot \mathrm{m}^{-3}$ 和 $\left.3.77 \mathrm{~m} \cdot \mathrm{g}^{-1} ; 3\right)$ 细根生物量、 $R L D$ 和 $S R L$ 与土壤水分、温 度和有效 $\mathrm{N}$ 存在不同程度的相关性。从单因子分析来看, 土壤水分和有效 $\mathrm{N}$ 对细根的影响明显大于温度, 对活根 的影响大于死根。由于土壤资源有效性的季节变化, 使得 $\mathrm{C}$ 的地下分配格局发生改变。各土层细根与有效性资源 之间的相关性反映了细根功能季节性差异。细根 (生物量、RLD 和 $S R L$ ) 的季节动态 (58\% 73\% 的变异) 主要由土 壤资源有效性的季节变化引起。

关键词 落叶松 细根生物量 根长密度 比根长 土壤资源有效性 季节动态

\section{SEASONAL DYNAMICS OF FINE ROOT BIOMASS, ROOT LENGTH DENSITY, SPECIFIC ROOT LENGTH AND SOIL RESOURCE AVAILABILITY IN A LARIX GMELINI PLANTATION}

\author{
CHENG Yun-Huan ${ }^{1}$ HAN You-Zhi ${ }^{1,2}$ WANG Qing-Cheng ${ }^{1}$ and WANG Zheng-Quan ${ }^{1 *}$ \\ (1 School of Forestry, Northeast Forestry University, Harbin, 150040, China) \\ (2 School of Forestry, Shanxi Agricultural University, Taigu, Shanxi 030801, China)
}

\begin{abstract}
Fine root turnover is a major pathway for carbon and nutrient cycling in terrestrial ecosystems and most likely is sensitive to many global change factors. Despite its importance in plant $\mathrm{C}$ allocation, nutrient cycling dynamics and the tremendous research efforts that have been made in the past, our understanding of fine root turnover remains limited, because dynamic fine root processes associated with soil resource availability still remains poorly understood. Soil moisture, temperature and available nitrogen are the most important soil resources that impact fine root growth and mortality at both the individual root branch and ecosystem level. In temperate forest ecosystems, seasonal changes of soil resource availability will alter the pattern of carbon allocation to below ground; therefore, fine root biomass, root length density $(R L D)$ and specific root length ( $S R L$ ) vary during the growing season. Studying seasonal changes of fine root biomass, $R L D$ and $S R L$ associated with soil resource availability will help us understand the mechanistic controls of carbon to fine root longevity and turnover.

The objective of this study was to understand whether seasonal variations of fine root biomass, $R L D$ and $S R L$ were associated with soil resource availability, such as moisture, temperature and nitrogen, and to understand how these soil components impacted fine root dynamics in a Larix gmelini plantation. We used a soil coring method to obtain fine root ( $\leqslant 2 \mathrm{~mm}$ in diameter) samples every month from May to October in 2002 from a 17 year old Larix gmelini plantation in Maoershan Experiment Station, Northeast Forestry University. Seventytwo soil cores (Inside diameter $60 \mathrm{~mm}$; depth intervals: $0-10,11-20$, and $21-30 \mathrm{~cm}$ ) were sampled ran-
\end{abstract}


domly from three replicate $25 \mathrm{~m} \times 30 \mathrm{~m}$ plots to estimate fine root biomass (live and dead), and calculate root length density $(R L D)$ and specific root length $(S R L)$. Soil moisture, temperature, and nitrogen (ammonia and nitrate) at the three depth intervals also were analyzed in the plots.

The results showed that the average standing fine root biomass (live and dead) was $189.1 \mathrm{~g} \cdot \mathrm{m}^{-2} \cdot \mathrm{a}^{-1}$, and $95.4 \mathrm{~g} \cdot \mathrm{m}^{-2} \cdot \mathrm{a}^{-1}(50 \%)$ was distributed in the surface soil layer $(0-10 \mathrm{~cm}), 61.5 \mathrm{~g} \cdot \mathrm{m}^{-2} \cdot \mathrm{a}^{-1}(33 \%)$ and $32.2 \mathrm{~g} \cdot \mathrm{m}^{-2} \cdot \mathrm{a}^{-1}(17 \%)$ were in middle $(11-20 \mathrm{~cm})$ and deep layer $(21-30 \mathrm{~cm})$, respectively. Live and dead fine root biomass was the highest from May to July and in September, but lower in August and October. The live fine root biomass decreased and dead biomass increased during the growing season. Mean $R L D$ (7 $\left.411.56 \mathrm{~m} \cdot \mathrm{m}^{-3} \cdot \mathrm{a}^{-1}\right)$ and $\operatorname{SRL}\left(10.83 \mathrm{~m} \cdot \mathrm{g}^{-1} \cdot \mathrm{a}^{-1}\right)$ in the surface layer were greater than the $R L D$ $\left(1474.68 \mathrm{~m} \cdot \mathrm{m}^{-3} \cdot \mathrm{a}^{-1}\right)$ and $S R L\left(8.56 \mathrm{~m} \cdot \mathrm{g}^{-1} \cdot \mathrm{a}^{-1}\right)$ in the deep soil layer. Root length density and $S R L$ in May were the highest $\left(10621.45 \mathrm{~m} \cdot \mathrm{m}^{-3}\right.$ and $\left.14.83 \mathrm{~m} \cdot \mathrm{g}^{-1}\right)$ as compared to other months, and $R L D$ was the lowest in September $\left(2198.20 \mathrm{~m} \cdot \mathrm{m}^{-3}\right)$ and $S R L$ the lowest in October $\left(3.77 \mathrm{~m}^{\bullet} \mathrm{g}^{-1}\right)$. Seasonal dynamics of fine root biomass, $R L D$ and $S R L$ had a close relationship with changes in soil moisture and nitrogen availability, and, to a lesser extent, temperature, as determined by regression analysis. Fine roots in the upper soil layer have a function of absorbing water and nutrients, while the main function of fine roots in the deeper soil may be water uptake rather than nutrient acquisition. Therefore, carbon allocation to roots in the upper soil layer and deeper soil layers was different. Multiple regression analysis showed that variation in soil resource availability can explain $71 \%-73 \%$ of the seasonal variation of $R L D$ and $S R L$ and $58 \%$ of the variation in fine root biomass. These results suggest a greater metabolic activity of fine roots living in soils with high resource availability, resulting in an increased allocation of carbohydrates to fine roots in resource rich soils but lower allocation to roots in soils with lower resource availability.

Key words Larix gmelini, Fine root biomass, Root length density, Specific root length, Soil resource availability, Seasonal dynamics

根系生物量是 $\mathrm{C}$ 向地下分配的结果。在生长 季中, 粗根的生物量变化很小, 细根 (直径 $\leqslant 2 \mathrm{~mm}$ ) 由于不断生长和死亡 (即周转), 变化较大 (温达志 等, 1999; 李凌浩等, 1998; Vogt et al., 1996; Fahey \& Hughes, 1994)。因此, 细根生物量季节动态可以反 映 C 的地下分配格局 (Gill \& Jackson, 2000; Bloomfield et al. 1996)。为了获取充足的养分和水分, 树木必 须维持一定的细根生物量 (Hendrick \& Pregitzer, 1992), 这种能力既受树冠同化 C 总量的调节 (Körner, 2003; Shipley \& Meziane, 2002), 也受土壤资 源(如水分、温度和养分等)有效性的影响 (Domisch et al., 2002; Bloomfield et al., 1996; Gower et al., 1992)。许多研究表明, 当土壤资源有效性增加时, C 向地下分配增多, 促进细根生长和生物量的积累, 细 根吸收养分和水分的能力增强 (King et al ., 2002; Gaudinski et al.,2001; Burton et al.,2000; Steele et $a l ., 1997)$ 。由于土壤资源有效性具有明显的季节 变化 (Pregitzer et al ., 2002), 导致细根生物量的季节 性波动(张小全, 2001; Vogt et al., 1996), 两者之间 的变化关系对森林生态系统 $\mathrm{C}$ 的地下分配格局和 细根周转具有重要的影响 (Lauenroth \& Gill, 2003;
土壤资源有效性不但具有季节上的变化, 还具 有垂直分布的差异 (Canadell et al., 1996), 使得不 同季节, 甚至同一季节各个层次细根的结构和功能 可能发生转变 (Hutchings \& John, 2003; Pregitzer et $a l$, , 2000)。因此, 对细根的研究, 除了生物量外, 还 应当考虑反映土壤资源有效性的根长密度 (Root length density; $R L D$ ) 和反映细根生理功能的比根长 (Specific root length; SRL) 这两个重要指标。但是, 以 往大多数研究仅注重细根生物量变化 ( López et al., 2001 ), 考虑土壤资源有效性对细根的影响时, 也仅 仅比较生物量年平均值有何差异 (Vogt et al ., 1996; Fahey \& Hughes, 1994), 忽视了季节变化过程中细根 功能的转变。对此, 我们以落叶松 (Larix gmelini) 人 工林为研究对象, 探讨: 1) 不同土层细根生物量、根 长密度和比根长的季节动态; 2) 这些动态与土壤水 分、温度和 $\mathrm{N}$ 素有效性的关系。目的是从细根的环 境和功能方面解释细根生物量季节变化的原因, 为 深入了解细根周转过程提供参考。

\section{1 研究地点和研究方法}

\section{1 研究林分概况}

研究地点位于黑龙江省尚志市东北林业大学帽 
儿山林场尖砬沟森林培育实验站 $\left(127^{\circ} 30^{\prime} \sim 127^{\circ} 34^{\prime}\right.$ $\left.\mathrm{E}, 45^{\circ} 21^{\prime} \sim 45^{\circ} 25^{\prime} \mathrm{N}\right)$ 。该地区属温带大陆性季风气 候。年均气温 $2.8{ }^{\circ} \mathrm{C}, 1$ 月平均温度 $-19.6{ }^{\circ} \mathrm{C}$; 7 月 平均气温 $20.9{ }^{\circ} \mathrm{C}$, 年平均降水量 $723 \mathrm{~mm}$, 年平均蒸 发量 $1094 \mathrm{~mm}$, 无霜期 $120 \sim 140 \mathrm{~d}, \geqslant 10{ }^{\circ} \mathrm{C}$ 的积温 $2526{ }^{\circ} \mathrm{C}$ 。样地设于山坡中上部, 海拔 $443 \mathrm{~m}$, 坡向 $\mathrm{NW} 10^{\circ}$, 坡度 $15^{\circ}$ 左右, 土壤为暗棕壤, 平均土层厚度 在 $40 \mathrm{~cm}$ 左右。林分为 17 年生落叶松人工纯林, 株 行距 $1.5 \mathrm{~m} \times 2.0 \mathrm{~m}$, 样地面积为 $25 \mathrm{~m} \times 30 \mathrm{~m}$ (3 次重 复), 目前落叶松平均树高 $10.3 \mathrm{~m}$, 平均胸径 10.6 $\mathrm{cm}$ 。

\section{2 研究方法}

\subsection{1 取样和根系分级}

2002 年从 5 月到 10 月生长季内, 每月中下旬在 样地内随机选择 24 个样点, 每样点用内径为 $60 \mathrm{~mm}$ 的土钻分 3 层钻取土芯样品, 每层 $10 \mathrm{~cm}$, 每次取样 72 个。将取出的土芯样品编号后放入塑料袋内, 在 实验站用 40 目 $(0.42 \mathrm{~mm})$ 篮网在流水中冲洗, 将洗 净后的根系迅速放入塑料袋、低温冷冻保存。样品 用冰块冷冻带回实验室, 按根系直径分为 3 级: 细根 直径 $\leqslant 2 \mathrm{~mm}$, 中根直径 $2 \sim 5 \mathrm{~mm}$, 粗根直径 $>5 \mathrm{~mm}$ （Smit et al.,1999）。并根据外形、颜色、弹性等区别 死根和活根(黄建辉等, 1999; Wang et al ., 1995)。

\section{$\mathbf{1 . 2} .2$ 生物量、根长密度及比根长计算}

每个月将分级后直径 $\leqslant 2 \mathrm{~mm}$ 的活根, 取 $2 / 3$ 左 右(生物量)在 $65{ }^{\circ} \mathrm{C}$ 烘干至恒重, 在电子天平 (精度 $0.001 \mathrm{~g}$ ) 上称量其干重。同时, 另 $1 / 3$ 左右样品放在 有网格纸的玻璃板上, 用不锈钢镊子夹住两端拉直, 实测长度 (精确到 $0.1 \mathrm{~mm}$ ), 然后烘干称重, 用于计 算各土芯样品的比根长 $(S R L)$ 。最后将两部分烘干 根系样品相加, 得到每一层土芯样品的生物量(死根 和直径 $>2 \mathrm{~mm}$ 的其它活根直接烘干称重)。根据每 层样品的根长和生物量计算各层的比根长 (SRL), 根据根长和 $10 \mathrm{~cm}$ 土芯体积计算根长密度 $(R L D)$ 。 最后计算林地每层 (24 个土芯样品) 各参数的平均 值, 并换算成单位面积上的生物量 $\left(\mathrm{g} \cdot \mathrm{m}^{-2}\right)$ 、比根长 $\left(\mathrm{m} \cdot \mathrm{g}^{-1}\right)$ 和根长密度 $\left(\mathrm{m} \cdot \mathrm{m}^{-3}\right)$ 。将 3 个土层 $(0 \sim 10$ $\mathrm{cm}, 11 \sim 20 \mathrm{~cm}, 21 \sim 30 \mathrm{~cm}$ ) 的生物量、根长密度和比 根长相加和平均, 得到林地每月的平均值。

\subsection{3 土壤速效氮、土壤温度、土壤含水量的测定}

每个月在取土芯样品同时, 将各个土芯样品分 别用 20 目 $(0.84 \mathrm{~mm}$ )笁网小心篮出部分土壤(约 80 $\mathrm{g})$, 迅速密封放入冰箱冷冻, 然后带回实验室分析土 壤硝态氮和铵态氮含量。硝态氮采用酚二磺酸比色
法, 铵态氮采用 $2 \mathrm{~mol} \cdot \mathrm{L}^{-1} \mathrm{KCl}$ 浸提-靛酚蓝比色法。 本研究硝态氮和铵态氮为 24 个土芯样品平均值。 在分析土壤 $\mathrm{N}$ 素的同时,测定土壤含水量。土壤温 度数据采用帽儿山生态站 (土壤 $5 、 15$ 和 $25 \mathrm{~cm}$ 处) 6 年观测平均值。

\section{3 数据分析}

对获得的细根数据和土壤数据, 采用方差分析 方法, 检验不同季节、不同土层之间细根生物量、根 长密度和比根长的差异。用回归分析方法, 分析细 根生物量、根长密度、比根长季节动态与土壤水分、 温度和有效 $\mathrm{N}$ 的关系。然后采用逐步回归分析方 法, 分析土壤水分、温度和有效 $\mathrm{N}$ 对细根动态的综 合影响。

\section{2 研究结果}

\section{1 细根生物量动态}

落叶松细根 (直径 $\leqslant 2 \mathrm{~mm}$ ) 生物量具有明显的 垂直分布上的差异和季节变化。它的年平均生物量 (活根 + 死根) 为 $189.1 \mathrm{~g} \cdot \mathrm{m}^{-2} \cdot \mathrm{a}^{-1}$, 其中 $50 \%(95.4$ $\left.\mathrm{g} \cdot \mathrm{m}^{-2} \cdot \mathrm{a}^{-1}\right)$ 分布在表层 $(0 \sim 10 \mathrm{~cm}), 33 \%$ 分布在 11 $\sim 20 \mathrm{~cm}\left(61.5 \mathrm{~g} \cdot \mathrm{m}^{-2} \cdot \mathrm{a}^{-1}\right)$ 土层。Schenk 和 Jackson (2002) 认为, 这种随土层加深细根生物量分布逐渐 减少的方式主要与有效性土壤资源的分布格局有 关。0 30 cm 土层的总细根生物量 (活根 + 死根) 在 5 7 月以及 9 月较高, 范围在 $194.6 \sim 214.4$ $\mathrm{g} \cdot \mathrm{m}^{-2}$ 之间, 8 和 10 月较低, 分别为 $155.9 \mathrm{~g} \cdot \mathrm{m}^{-2}$ 和 $157.1 \mathrm{~g} \cdot \mathrm{m}^{-2}$ (图 1a)。在土层 $0 \sim 10 \mathrm{~cm}$ 和 $11 \sim 20 \mathrm{~cm}$ 中, 细根生物量的变化特征与总生物量相似, 而底层 (21 30 cm) 生物量在整个生长季逐渐下降。虽然 总细根生物量具有季节变化特点, 但各月之间无显 著差异 $(p>0.05)$ 。

在区分活根和死根后, 细根生物量季节变化差 别明显(图 $1 b 、 c$ )。春季 ( 5 月) 和初夏 ( 6 月)活根生 物量最高 ( 177.8 和 $170.1 \mathrm{~g} \cdot \mathrm{m}^{-2}$ ), 死根生物量最低 (36.6 和 $\left.32.5 \mathrm{~g} \cdot \mathrm{m}^{-2}\right)$, 夏季和秋季, 活根生物量逐 渐减少 $\left(9 \sim 10\right.$ 月平均为 $\left.87.1 \mathrm{~g} \cdot \mathrm{m}^{-2}\right)$, 而死根生物 量逐渐增加 $\left(9 \sim 10\right.$ 月平均为 $\left.88.1 \mathrm{~g} \cdot \mathrm{m}^{-2}\right)$, 表明细 根不断周转 (Bloomfield et al., 1996; Hendrick \& Pregitzer, 1993)。方差分析显示, 春季活根和死根生物 量与夏季、秋季有明显差异 $(p<0.05)$ 。不同层次活 根和死根的生物量季节变化与相应总生物量 $(0 \sim 30$ $\mathrm{cm})$ 的变化格局相似, 但表层 $(0 \sim 10 \mathrm{~cm})$ 的季节变化 大于底层。 

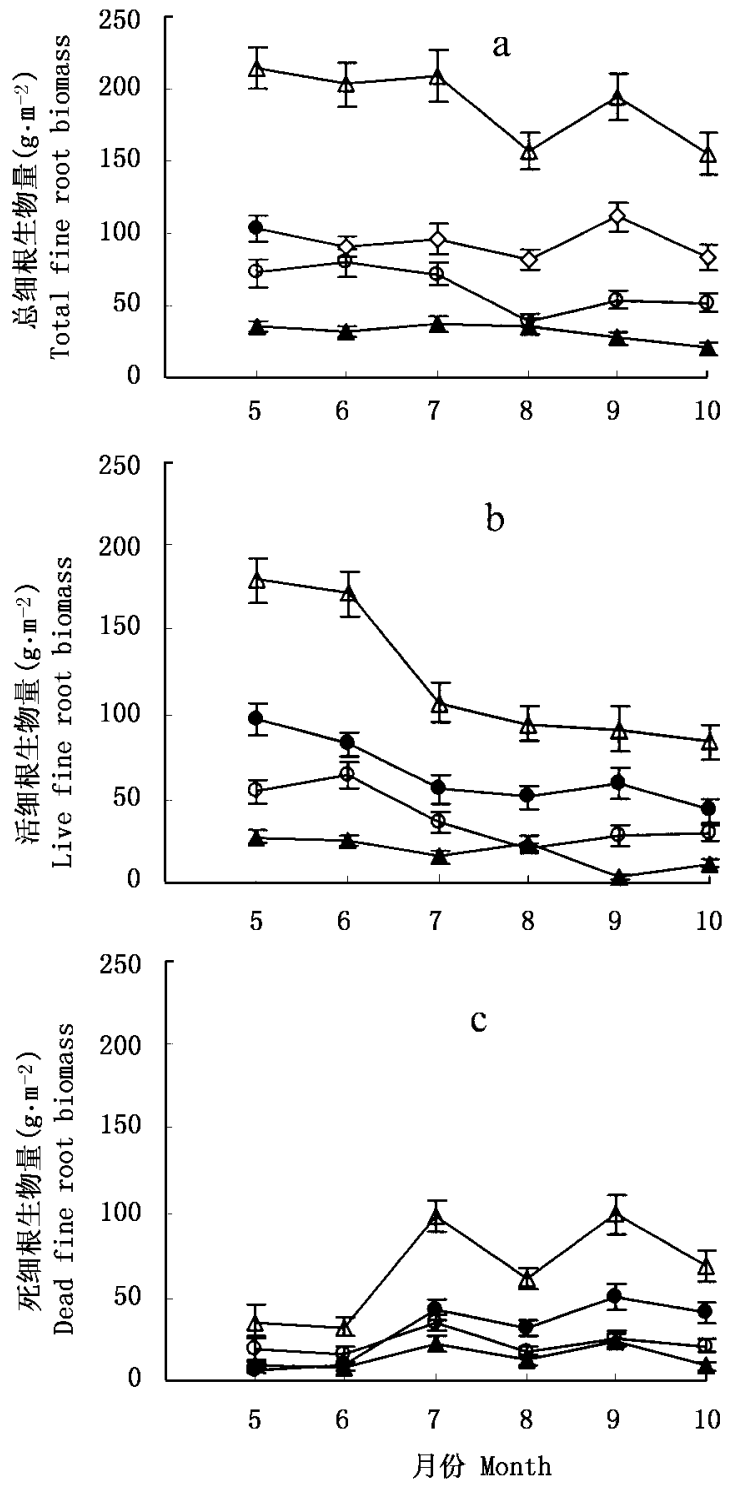

图 1 落叶松人工林 $(17$ 年) 不同土层总细根 $(\mathrm{a})$ 、活细根 $(\mathrm{b})$ 及 死细根 $(\mathrm{c})$ (直径 $\leqslant 2 \mathrm{~mm}$ ) 生物量动态

Fig. 1 Dynamics of total (a), live (b) and dead (c) fine root biomass ( $\leqslant 2 \mathrm{~mm}$ in diameter) of larch plantation( 17 years) in different soil depths through months

土层 Soil depth: $\triangle 0 \sim 30 \mathrm{~cm} \quad 0 \sim 10 \mathrm{~cm} \quad \bigcirc 11 \sim 20 \mathrm{~cm}$ $21 \sim 30 \mathrm{~cm}$

图 2、图 3 图例同图 1 The legend of Fig.2, Fig.3 are the same as that of Fig. 1

\section{2 根长密度 $(R L D)$ 和比根长 $(S R L)$ 动态}

$R L D$ 和 $S R L$ 大小与土壤资源的有效性相关 (Burton et al., 2000; Eissenstat, 1991; Burke et al., 1991)。年平均统计结果显示, 土壤表层 $(0 \sim 10 \mathrm{~cm})$ 具有较高的 $R L D\left(7411.56 \mathrm{~m} \cdot \mathrm{m}^{-3} \cdot \mathrm{a}^{-1}\right)$ 和 $S R L$ $\left(10.83 \mathrm{~m}^{\bullet} \mathrm{g}^{-1} \cdot \mathrm{a}^{-1}\right)$, 说明细根分布多且直径较细, 可以提高吸收养分的效率, 而底层 $(21 \sim 30 \mathrm{~cm})$ 最低 (1 $\left.474.68 \mathrm{~m} \cdot \mathrm{m}^{-3} \cdot \mathrm{a}^{-1}, 8.56 \mathrm{~m} \cdot \mathrm{g}^{-1} \cdot \mathrm{a}^{-1}\right)$, 意味着根
量少且直径相对较粗 (Smit et al., 1999), 底层的细 根可能主要以吸收水分为主 (Burton et al .,2000)。 另外, 不同季节, $R L D$ 和 $S R L$ 具有较大差异(图 2, 图 3 )。春季 (5月)树木开始生长时, 对水分和养分的 需求旺盛, 总 $R L D$ 和 $S R L$ 最高, 分别 10621.45 $\mathrm{m} \cdot \mathrm{m}^{-3}$ 和 $14.83 \mathrm{~m} \cdot \mathrm{g}^{-1}$, 生长季内逐渐下降, 到秋季 (9月)树木生长结束后达到最低值, $2198.20 \mathrm{~m} \cdot \mathrm{m}^{-3}$ 和 $3.77 \mathrm{~m} \cdot \mathrm{g}^{-1}$ 。这种变化规律也存在于不同土层 中,而且各个土层之间以及同一土层不同季节之间 的 $R L D$ 具有显著差异 $(p<0.05), S R L$ 除 $7 、 8$ 和 10 月外也差异显著 $(p<0.05)$ 。

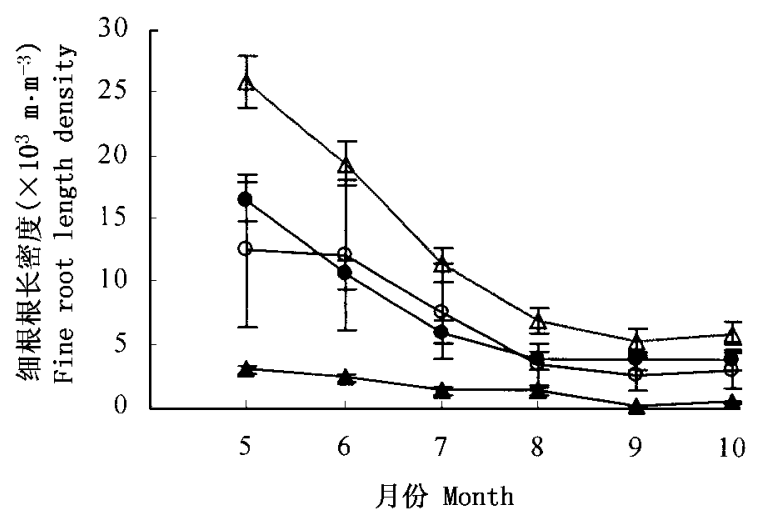

图 2 落叶松人工林 (17 年) 不同土层细根 (直径 $\leqslant 2 \mathrm{~mm}$ ) 根长密度 $(R L D)$ 动态

Fig.2 Dynamics of root length density ( $R L D$ ) of larch in different soil depths through months

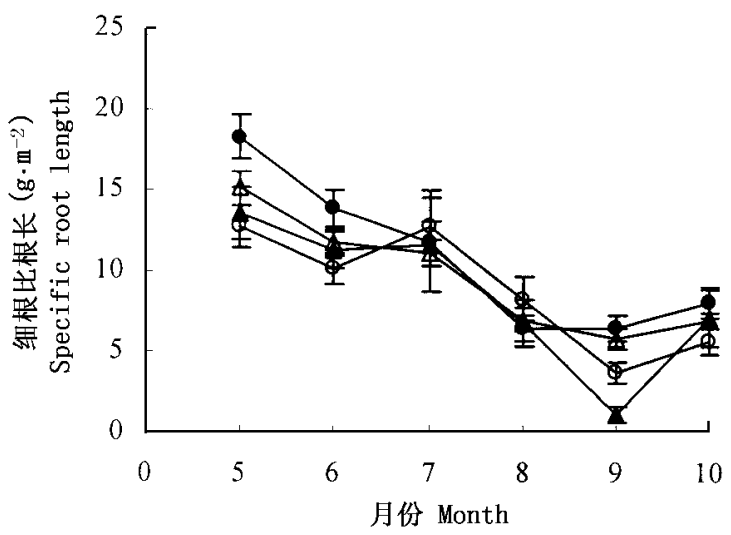

图 3 落叶松人工林 (17 年)不同土层根 (直径 $\leqslant 2 \mathrm{~mm}$ ) 比根长 $(S R L)$ 动态

Fig.3 Dynamics of specific root length ( $S R L$ ) of larch in different soil depths through months

2.3 细根动态与土壤水分、温度和速效 $\mathrm{N}$ 的关系 温带地区土壤水分、温度和有效 $\mathrm{N}$ 具有明显的 季节特点, 直接影响细根的生命过程 (Pregitzer et 
al .,2000; Usman et al ., 1999)。一元回归分析表明, 落叶松细根生物量、 $R L D$ 和 $S R L$ 与土壤水分、温度 和有效 $\mathrm{N}$ 具有不同程度的相关性 (表 1), 总体上土 壤水分和有效 $\mathrm{N}$ 对细根的影响明显大于温度。土 壤水分与死根生物量的相关性 $(r=-0.78)$ 高于活 根 $(r=0.33)$, 但是上层 $(0 \sim 20 \mathrm{~cm})$ 活细根生物量随 水分增加而增加 $(r=0.31 \sim 0.35)$, 死细根则相反 $(r$ $=-0.69 \sim-0.70)$ 。底层 $(21 \sim 30 \mathrm{~cm})$ 的水分的增 加会引起活细根生物量显著减少 $(r=-0.81 ; p<$ $0.05)$ 和死细根生物量的增多 $(r=0.61)$ 。当土壤含 水量从表层 $(0 \sim 10 \mathrm{~cm})$ 的 $69 \%$ 减少到亚表层 $(11 \sim$ $20 \mathrm{~cm}$ ) 的 $50 \%$ 时, $R L D$ 和 $S R L$ 与水分的相关性也在 降低 (分别为 $r=0.50 \sim 0.39 ; r=0.98 \sim 0.42$ ), 在底 层为显著的负相关 (分别为 $r=-0.90 ; r=-0.90$, $p<0.05)$ 。说明土壤水分有效性的提高, 引起上层 细根密度增加和直径减小, 以及底层密度降低和直 径增粗。

落叶松林地土壤表层 $(0 \sim 10 \mathrm{~cm})$ 总有效 $\mathrm{N}$ (铵 态 $\mathrm{N}+$ 硝态 $\mathrm{N}$ ) 含量最高 $\left(16.8 \mathrm{mg} \cdot \mathrm{kg}^{-1}\right)$, 亚表层 $(11$ $\sim 20 \mathrm{~cm}$ ) 次之 $\left(11.8 \mathrm{mg} \cdot \mathrm{kg}^{-1}\right)$, 底层 $(21 \sim 30 \mathrm{~cm})$ 最 少 (7.9 $\left.\mathrm{mg} \cdot \mathrm{kg}^{-1}\right)$ 。对于比较肥沃的土壤(如表层, $0 \sim 10 \mathrm{~cm}$ ), 随着土壤 $\mathrm{N}$ 有效性增加, 生物量( 总细 根: $r=-0.85, p<0.05$; 活细根: $r=-0.39) 、 R L D$ $(r=-0.20)$ 下降(表 1), 对死细根 $(r=0.08)$ 和 $S R L$ $(r=0.04$ ) 影响很小。较贫痊的土壤层(如亚表层, 11 20 cm), $\mathrm{N}$ 有效性增加促进细根生物量( 总细 根: $r=0.78$; 活细根: $r=0.91, p<0.05) 、 R L D(r=$ $0.74)$ 和 $S R L(r=0.23)$ 的提高。这既反映了土壤有 效 $\mathrm{N}$ 含量不同情况下细根 $\mathrm{C}$ 的分配格局, 又反映了 细根吸收养分能力的差异 (Nadelhoffer, 2000; Gower et al .,1992)。两种形态的 $\mathrm{N}$ 对于表层和亚表层细 根生物量、 $R L D$ 和 $S R L$ 影响与总有效 $\mathrm{N}$ 的影响一 致, 只是对细根生长的作用小于总有效 $\mathrm{N}$ 的作用 （表 1)，从 3 层平均看, 铵态 $\mathrm{N}$ 对细根生长的影响小 于硝态 $\mathrm{N}$ 。但上述关系在底层 $(21 \sim 30 \mathrm{~cm}$ ) 没表现 出来, 可能与不同层次细根的功能差异相关。

细根的生长是土壤水分、温度和养分综合作用 的结果( Pregitzer et al.,2000), 既表现在不同的土壤 层次之间, 又表现在平均水平上。采用土壤水分、温 度和有效 $\mathrm{N}$ 进行逐步线性回归分析表明(表 1),这 3 个因子综合, 对落叶松细根生物量、 $R L D$ 和 $S R L$ 有 比较大的影响。可以解释总细根生物量、 $R L D$ 和 $S R L$ 变异的 $71 \% \sim 73 \%$, 解释活细根生物量变异的 $58 \%$, 也就是说落叶松细根的季节动态 ( $58 \% \sim 73 \%$
的变异)主要是由土壤资源有效性的季节变化引起 的。

\section{3 讨 论}

温带森林由于受气候因子控制, 年地上生长过 程和地下生长过程具有明显的季节性 (Pregitzer et al .,2000; Fahey \& Hughes, 1994)。研究表明(图 1～ 3 ), 落叶松细根生物量、 $R L D$ 和 SRL 均表现出明显 的季节特点。春季 ( 5 月) 是树木开始萌动和生长的 季节, 为了满足叶和嫩枝的发育, 需要根系提供大量 养分和水分 (Canadell et al ., 1996; Hendrick \& Pregizer, 1996)。这时土壤解冻, 水分含量较高 (平均 $56 \%$ ), 但温度 (平均 $9.4{ }^{\circ} \mathrm{C}$ )、微生物的矿化活动以 及有效 $\mathrm{N}\left(0 \sim 20 \mathrm{~cm}\right.$ 平均 $\left.13.9 \mathrm{mg} \cdot \mathrm{g}^{-1}\right)$ 含量均较低, 这样就迫使根系持有较高的生物量才能满足春季生 长的需求。因此, 春季 ( 5 月) 活细根生物量最高 $\left(177.8 \mathrm{~g} \cdot \mathrm{m}^{-2}\right)$ (图 $1 \mathrm{~b}$ ), 密度 $(R L D)$ 最大 $(10621.45$ $\left.\mathrm{m} \cdot \mathrm{m}^{-3}\right)$, 直径 $(S R L)$ 也最细 $\left(14.83 \mathrm{~m} \cdot \mathrm{g}^{-1}\right)$, 以提高 吸收养分和水分的效率(Eissenstat \& Yanai，1997)。

许多研究表明, 温带落叶树种细根生长先于叶 的生长 (Pregitzer et al., 2000; Hendrick \& Pregitzer, 1996; Eissenstat \& van Rees, 1994)。春季( 5 月)展叶 之前, 细根生长所需的 $\mathrm{C}$ 来自于根系上一年储存的 碳水化合物, 展叶后来自于新叶固定的碳水化合物 (Pregitzer，2003)。由于后一部分 C 分配到细根的有 限, 细根很快消耗原来储存的碳水化合物, 致使新生 的细根在随后一段时间内陆续死亡 (Anderson et $a l ., 2003$ )。夏季 (7 月) 虽然土壤水分充足 (平均 $45 \%$ )、土壤温度(平均 $18.9{ }^{\circ} \mathrm{C}$ ) 和有效 $\mathrm{N}$ 含量 $(0 \sim$ $20 \mathrm{~cm}$ 平均 $\left.15.7 \mathrm{mg} \cdot \mathrm{g}^{-1}\right)$ 也较高, 但活细根生物量的 减少 $\left(105.9 \mathrm{~g} \cdot \mathrm{m}^{-2}\right.$, 比 5 月减少 $40 \%$, 图 $\left.1 \mathrm{~b}\right)$, 死细根 的生物量增加 $\left(103.1 \mathrm{~g} \cdot \mathrm{m}^{-2}\right.$, 比 5 月增加 $181 \%$, 图 1c), $R L D$ 下降 ( $5036.64 \mathrm{~m} \cdot \mathrm{m}^{-3}$, 比 5 月减少 $53 \%$ )、 $S R L$ 减小 $\left(12.03 \mathrm{~m}^{\bullet} \mathrm{g}^{-1}\right.$, 比 5 月减少 $\left.19 \%\right)$ 。Pregitzer (2003) 认为这是因为夏季 $\mathrm{C}$ 分配格局发生改变, 即 使土壤资源有效性利于细根生长及养分和水分吸 收, 相当一部分 $\mathrm{C}$ 还是分配到地上的枝条和茎中。 在夏季树冠发育完全后, 细根吸收养分和水分与消 耗的 $\mathrm{C}$ 之间保持一个相对的动态平衡 (Hendrick \& Pregitzer, 1996)。活细根和 $R L D$ 的减少与春季生长 的细根的死亡有关, 直径增粗 ( SRL 下降)与新生长 的细根有关, 因为在夏季土壤资源有效性达到最高 (Pregitzer et al.,2000), 新生长的细根具有较低的 SRL (Majdi, 2001; Espeleta \& Donovan, 2002)。秋季, 
表 1 落叶松人工林细根 (直径 $\leqslant \mathbf{2} \mathbf{m m}$ ) 生物量、根长密度 $(\boldsymbol{R} \boldsymbol{L D})$ 和比根长 $(\boldsymbol{S R} \boldsymbol{L})$ 与土壤水分、温度、硝态氮、氨态氮的相关关系 Table 1 Correlation coefficients of fine root $(\leqslant 2 \mathrm{~mm}$ in diameter $)$ biomass, root length density $(R L D)$ and specific root length

$(S R L)$ with soil moisture, temperature, $\mathrm{NH}_{4}^{+}$and $\mathrm{NO}_{3}^{-}$in larch plantation

\begin{tabular}{|c|c|c|c|c|c|c|c|}
\hline \multirow{2}{*}{$\begin{array}{c}\text { 土层 } \\
\text { Soil depth }(\mathrm{cm})\end{array}$} & \multicolumn{7}{|c|}{ 相关系数 Correlation coefficients $(r)$} \\
\hline & $r_{\mathrm{w}}$ & $r_{\mathrm{T}}$ & $r_{\mathrm{NH}_{4}}$ & $r_{\mathrm{NO}_{3}}$ & $r_{\mathrm{NH}_{4}}-\mathrm{NO}_{3}$ & $r_{\mathrm{W}+\mathrm{T}}^{2}$ & $r_{\mathrm{W}+\mathrm{T}+\mathrm{N}}^{2}$ \\
\hline \multicolumn{8}{|c|}{ 总细根生物量 Total fine root biomass } \\
\hline $0 \sim 10$ & -0.37 & 0.001 & 0.36 & 0.33 & $-0.85^{*}$ & 0.40 & 0.79 \\
\hline $11 \sim 20$ & 0.01 & 0.03 & 0.48 & 0.52 & 0.78 & 0.04 & 0.61 \\
\hline $21 \sim 30$ & -0.44 & 0.47 & 0.67 & 0.07 & -0.59 & $0.89^{*}$ & $0.82^{*}$ \\
\hline $0 \sim 30$ & -0.44 & 0.16 & 0.20 & 0.61 & 0.21 & 0.16 & 0.71 \\
\hline \multicolumn{8}{|c|}{ 活细根生物量 Live fine root biomass } \\
\hline $0 \sim 10$ & 0.31 & 0.04 & -0.19 & 0.13 & -0.39 & 0.11 & 0.54 \\
\hline $11 \sim 20$ & 0.35 & -0.16 & 0.56 & 0.62 & $0.91^{*}$ & 0.10 & $0.88^{*}$ \\
\hline $21 \sim 30$ & $-0.81^{*}$ & 0.07 & -0.13 & 0.06 & 0.07 & 0.79 & 0.79 \\
\hline $0 \sim 30$ & 0.33 & 0.08 & 0.04 & 0.61 & 0.57 & 0.11 & 0.58 \\
\hline \multicolumn{8}{|c|}{ 死细根生物量 Dead fine root biomass } \\
\hline $0 \sim 10$ & -0.69 & 0.10 & 0.01 & 0.08 & 0.08 & 0.56 & 0.67 \\
\hline $11 \sim 20$ & -0.70 & 0.31 & 0.24 & 0.29 & -0.41 & 0.51 & 0.62 \\
\hline $21 \sim 30$ & 0.61 & 0.50 & 0.43 & 0.01 & -0.43 & 0.43 & 0.46 \\
\hline $0 \sim 30$ & -0.78 & 0.24 & 0.23 & 0.31 & -0.60 & 0.61 & 0.38 \\
\hline \multicolumn{8}{|c|}{ 活细根根密度 Root length density ( $R L D)$} \\
\hline $0 \sim 10$ & 0.50 & 0.05 & 0.10 & 0.07 & -0.20 & 0.25 & 0.52 \\
\hline $11 \sim 20$ & 0.39 & 0.05 & 0.36 & 0.59 & 0.74 & 0.15 & 0.68 \\
\hline $21 \sim 30$ & $-0.90^{*}$ & -0.20 & 0.18 & 0.30 & 0.10 & $0.89^{*}$ & $0.92^{*}$ \\
\hline $0 \sim 30$ & 0.36 & -0.14 & 0.02 & 0.60 & 0.49 & 0.13 & 0.73 \\
\hline \multicolumn{8}{|c|}{ 活细根比根长 Specific root length (SRL) } \\
\hline $0 \sim 10$ & $0.98^{*}$ & 0.01 & 0.01 & 0.01 & 0.04 & $0.96^{*}$ & $0.98^{*}$ \\
\hline $11 \sim 20$ & 0.42 & -0.21 & 0.09 & 0.20 & 0.23 & 0.28 & 0.33 \\
\hline $21 \sim 30$ & $-0.90^{*}$ & -0.15 & 0.09 & 0.01 & 0.08 & $0.92^{*}$ & $0.96^{*}$ \\
\hline $0 \sim 30$ & 0.30 & 0.05 & 0.03 & 0.55 & 0.43 & 0.10 & 0.73 \\
\hline
\end{tabular}

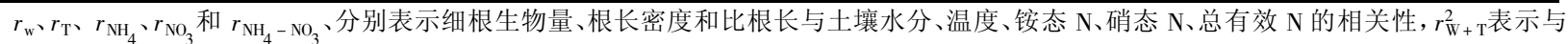
土壤水分和温度的相关性, $r_{\mathrm{W}+\mathrm{T}+\mathrm{N}}^{2}$ 表示与土壤水分、温度和总有效 $\mathrm{N}$ 的相关性, * 表示相关性显著, $p<0.05 \quad r_{\mathrm{w}} 、 r_{\mathrm{T}} 、 r_{\mathrm{NH}_{4}} 、 r_{\mathrm{NO}_{3}}$ and $r_{\mathrm{NH}_{4}-\mathrm{NO}_{3}}$ are correlation coefficients of fine root biomass, $R L D$ and $S R L$ with soil moisture, soil temperature, ammonia, nitrates and total nitrogen availability respectively, $r_{\mathrm{W}+\mathrm{T}}^{2}$ is multiple correlation coefficient of fine root biomass, $R L D$, SRL with soil resource (moisture and soil temperature availability, respectively. $r_{\mathrm{W}+\mathrm{T}+\mathrm{N}}^{2}$ is multiple correlation coefficient of fine root biomass, $R L D$, $S R L$ with soil resource (moisture, soil temperature and total nitrogen) availability, respectively, $* p<0.05$

随着气温逐渐降低,落叶松地上部分停止生长 $(8$ 月 末), 树冠逐渐衰老( 9 月), 对养分和水分的需求降 低。落叶后,有大量的光合产物 (以淀粉形式) 储存 在根系中 (主要是维持休眠前呼吸消耗以及来年春 季细根的生长), 秋季活细根生物量、 $R L D$ 和 $S R L$ 的 持续减少归结于细根的死亡、根组织密度增加以及 木质化。

土壤各层次之间不同时期细根动态既反映细根 功能转换, 又反映土壤资源有效性的变化 (Nepstad et al ., 1994; Schulze et al., 1996)。对落叶松的研究 表明 (表 1), 不同土壤层次细根的动态差异是由水 分和养分综合作用的结果。细根养分和水分的吸收 是同时进行的过程, 表层土壤 $\mathrm{N}$ 含量高 (水分充足 和温度适宜), 因而细根主要集结在表层内以获取养 分 $(\mathrm{N})$, 毫无疑问这需要投入大量的 $\mathrm{C}($ Burton et $a l ., 2000)$ ，同时也会消耗比其它层次更多的水分。
因此, 只有水分得到满足, 才能促进细根的生长 (生 物量、 $R L D$ 和 $S R L$ 与水分正相关)。养分和水分都 不足的亚表层, 细根的生长既需要水分, 也需要养分 (生物量、 $R L D$ 和 $S R L$ 分别与水分和 $\mathrm{N}$ 正相关)。但 是由于水分和 $\mathrm{N}$ 含量有限, $\mathrm{C}$ 的投入相对较少, 致使 亚表层的细根生物量(占 $33 \%$ ) 比表层(占 $50 \%$ ) 显 著降低。在贫痊的底层, 细根可吸收的 $\mathrm{N}$ 很少, $\mathrm{C}$ 的 投入很低, 细根的功能与其它两层之间也存在差异, 以吸收水分为主 (Burton et al ., 2000)。但水分相对 过多, 会导致细根死亡增加 $(r=0.60)$ 以及活根的显 著减少 (活细根生物量、 $R L D$ 和 $S R L$ 与水分显著负 相关)。

无论细根生物量、 $R L D$ 和 $S R L$ 如何变化, 都是 土壤资源的有效性与树木自身内在因子 (如 $\mathrm{C}$ 分配 格局) 综合作用的结果 (Burke et al., 1991)。随着土 壤资源有效性的改变, $\mathrm{C}$ 的分配格局、细根的生理功 
能也会发生变化 (Burton et al ., 2000), 这是树木适应 环境的一种策略 (Eissenstat \& van Rees, 1994; Canadell et al ., 1996)。

\section{4 结 论}

1) 落叶松细根 (直径 $\leqslant 2 \mathrm{~mm}$ ) 年平均生物量 (活 根 + 死根 $)$ 为 $189.1 \mathrm{~g} \cdot \mathrm{m}^{-2} \cdot \mathrm{a}^{-1}$, 其中 $50 \%(95.4$ $\left.\mathrm{g} \cdot \mathrm{m}^{-2} \cdot \mathrm{a}^{-1}\right)$ 分布在表层 $(0 \sim 10 \mathrm{~cm}), 33 \%(61.5$ $\left.\mathrm{g} \cdot \mathrm{m}^{-2} \cdot \mathrm{a}^{-1}\right)$ 分布在中层 $(11 \sim 20 \mathrm{~cm}), 17 \%(32.2$ $\left.\mathrm{g} \cdot \mathrm{m}^{-2} \cdot \mathrm{a}^{-1}\right)$ 分布在底层 $(21 \sim 30 \mathrm{~cm})$ 。活根和死根 生物量在 5 7 月以及 9 月较高, 8 和 10 月较低。从 春季 ( 5 月)到秋季 (10 月), 随着活细根生物量的减 少, 死细根生物量增加。

2) 土壤表层 $(0 \sim 10 \mathrm{~cm})$ 具有较高的 $R L D$ $\left(7411.56 \mathrm{~m} \cdot \mathrm{m}^{-3}\right)$ 和 $S R L\left(10.83 \mathrm{~m} \cdot \mathrm{g}^{-1}\right)$, 而底层 (21 $\sim 30 \mathrm{~cm})$ 最低 $\left(1474.68 \mathrm{~m} \cdot \mathrm{m}^{-3}, 8.56 \mathrm{~m} \cdot \mathrm{g}^{-1}\right)$ 。春季 (5月), 总 $R L D$ 和 $S R L$ 最高, 分别为 10621.45 $\mathrm{m} \cdot \mathrm{m}^{-3}$ 和 $14.83 \mathrm{~m} \cdot \mathrm{g}^{-1}$, 生长季内逐渐下降, 到秋季 (9) 月)树木生长结束后达到最低值, 分别为 2198.20 $\mathrm{m} \cdot \mathrm{m}^{-3}$ 和 $3.77 \mathrm{~m} \cdot \mathrm{g}^{-1}$ 。这种变化规律也存在于不同 土层中。

3)落叶松细根生物量、 $R L D$ 和 $S R L$ 与土壤水 分、温度和有效 $\mathrm{N}$ 具有不同程度的相关性。从单因 子分析来看, 土壤水分和有效 $\mathrm{N}$ 对细根的影响明显 大于温度, 对活根的影响大于死根。由于土壤资源 有效性差异, C 的地下分配格局发生改变。各土层 之间相关性和细根功能不同。表层细根吸收养分和 水分, 底层的细根可能以吸收水分为主。细根 (生物 量、 $R L D$ 和 $S R L)$ 的季节动态 ( $58 \% \sim 73 \%$ 的变异) 主 要是由土壤资源有效性的季节变化引起的。

\section{参 考 文 献}

Anderson LJ, Comas LH, Lakso AN, Eissenstat DM (2003) . Multiple risk factors in root survivorship: a four-year study in Concord grape. New Phytologist, 158, 489 - 501 .

Bloomfield JK, Vogt KA, Wargo PM (1996). Tree root turnover and Senescence. In: Waisel Y, Eshel A, Kaafkafi U eds. Plant Roots: the Hidden Half. 2nd edn. Marcel Dekker, New York, $363-381$.

Burke MK, Raynai DJ, Mrrchell MJ (1991). Soil nitrogen availability influences seasonal carbon allocation patterns in sugar maple (Acer saccharum). Canadian Journal of Forest Research, 22, $447-456$.

Burton AJ, Pregitzer KS, Hendrick RL (2000). Relationships between fine root dynamics and nitrogen availability in Michigan northern hardwood forest. Oecologia, 125, 389-399.

Canadell J, Jackson RB, Ehleringer JR, Mooney HA, Sala OE, Schulze ED (1996). Maximum rooting depth of vegetation types at the global scale. Oecologia, 108, 583-595.

Domisch T, Finér L, Lehto T (2002). Growth, carbohydrate and nutrient allocation of Scots pine seedlings after exposure to simulated low soil temperature in spring. Plant and Soil, 246, 75 86.

Eissenstat DM, van Rees KCJ (1994). The growth and function of pine roots. Ecological Bulletins, 43, 76 - 91 .

Eissenstat DM, Yanai RD (1997). The ecology of root lifespan. Advances in Ecological Research, 27, 2-59.

Eissenstat EM (1991). On the relationship between specific root length and the rate of root proliferation: a field study using citrus rootstocks. New Phytologist, 118, 63-68.

Espeleta JF, Donovan LA (2002). Fine root demography and morphology in response to soil resources availability among xeric and mesic sandhill tree species. Functional Ecology, 16,113-121.

Fahey TJ, Hughes JW (1994). Fine root dynamics in northern hardwood forest ecosystem, Hubbard Brook experimental forest, NH. Journal of Ecology, 82, 533 - 548 .

Gaudinski JB, Trumbore SE, Davidson EA, Cook AC, Markewitz D, Richter D (2001). The age of fine-root carbon in three forests of the eastern United States measured by radiocarbon. Oecologia, $129,420-429$.

Gill RA, Jackson RB (2000). Global patterns of root turnover for terrestrial ecosystems. New Phytologist, 147,13 - 31 .

Gower ST, Vogt KA, Grier CC (1992) . Carbon dynamics of Rocky Mountain Douglas-fir: influence of water and nutrient availability. Ecological Monographs, 62, $43-65$.

Hendrick RL, Pregitzer KS (1992). The demography of fine root in a northern hardwood forest. Ecology, 73, $1094-1104$.

Hendrick RL, Pregitzer KS (1993). Patterns of fine root mortality in two sugar maple forests. Nature, 361, 59-61.

Hendrick RL, Pregitzer KS (1996). Temporal and depth-related patterns of fine root dynamics in northern hardwood forests. Journal of Ecology, 84,167 - 176.

Huang JH (黄建辉), Han XG (韩兴国), Chen LZ (陈灵芝) (1999). Advances in the research of (fine) root biomass in forest ecosystems. Acta Ecologica Sinica (生态学报), 19, 270 277. (in Chinese with English abstract)

Hutchings MJ, John EA (2003). Distribution of roots in soil, and root foraging activity. In: Kroon HD, Visser EJW eds. Root Ecology. Springer-Verlag, New York, $61-83$.

King JS, Albaugh TJ, Allen HL, Buford M, Strain BR, Dougherty $\mathrm{P}$ (2002) . Below-ground carbon input to soil is controlled by nutrient availability and fine root dynamics in loblolly pine. New Phytologist, 154, 389 - 398.

Körner C (2003) . Carbon limitation in trees. Journal of Ecology, $91,4-17$.

Lauenroth WK, Gill R (2003). Turnover of root systems. In: Kroon HD, Visser EJW eds. Root Ecology. Springer-Verlag, New York, $61-83$.

Li LH (李凌浩), Ling P (林鹏), Xing XR (邢雪荣) (1998). Fine root biomass and production of Castanopsis eyrei forests in Wuyi Mountains. Chinese Journal of Applied Ecology (应用生态 学报), 9, 337 - 340. (in Chinese with English abstract)

López B, Sabat S, Gracia CA (2001). Annual and seasonal changes in fine root biomass of a Quercus ilex L. forest. Plant and Soil, 230, $125-134$. 
Majdi H (2001). Changes in fine root production and longevity in relation to water and nutrient availability in a Norway spruce stand in northern Sweden. Tree Physiology, 21, 1057 - 1061 .

Nadelhoffer KJ (2000). Research review: the potential effects of nitrogen deposition on fine-root production in forest ecosystems. New Phytologist, 147, 131 - 139.

Nepstad DC, de Carvalbo CR, Davidson EA, Jipp PH, Lefevre PA, Negreiros GH, da Silva ED, Stone TA, Trumbore SE, Vieira S (1994). The role of deep roots in the hydrological and carbon cycles of Amazonian forests and pastures. Nature, 372, $666-669$.

Pregitzer KS ( 2003 ). Woody plants, carbon allocation and fine roots. New Phytologist, 158,421 - 423 .

Pregitzer KS, King JS, Burton AJ (2000). Response of tree fine roots to temperature. New Phytologist, 147,105-115.

Pregizer KS, Deforest JL, Burton AJ, Allen MF, Ruess RW, Hendrich RL (2002). Fine root architecture of nine north American trees. Ecological Monographs, 72,293-309.

Schenk HJ, Jackson RB (2002) . The global biogeography of roots. Ecological Monographs, 72,311 - 328 .

Schulze ED, Bauer G, Buchmann N, Canadell J, Ehleringer JR, Jackson RB, Jobbagy E, Loreti J, Mooney HA, Oesterbeld M, Sala O ( 1996). Water availability, rooting depth, and vegetation zones along an aridity gradient in Patagonia. Oecologia, 108,503 -511 .

Shipley B, Meziane D (2002). The balanced-growth hypothesis and the allometry of leaf and root biomass allocation. Functional Ecology, 16,326-331.
Smit AL, George E, Groenwold J (1999). Root observations and measurements at (transparent) interfaces with soil. In: Smit AL, Bengough AG, Engels C, Noordwijk MV, Pellerin S, van de Geijn SC eds. Root Methods. Springer-Verlag, New York, 236 $-266$.

Steele SJ, Gower ST, Vogel JG, Norman JM (1997). Root mass, net primary production and turnover in aspen, jack pine and black spruce forests in Saskatchewan and Manitova Canada. Tree Physiology, 17,577 - 587 .

Usman S, Singh SP, Rawat YS (1999). Fine root production and turnover in two evergreen central Himalayan forests. Annals of Botany, 84,87-94.

Vogt KA, Vogt DJ, Palmiotto PA, Boon P, Hara JO', Absbjornsen H (1996). Review of root dynamics in forest ecosystems grouped by climate, climatic forest type and species. Plant and Soil, $187,159-219$.

Wang ZQ, Burch WH, Mou P, Jones RH, Mitchell RJ (1995). Accuracy of visible and ultraviolet light for estimation live root proportions with minirhizotrons. Ecology, 76,2330-2334.

Wen DZ (温达志), Wei P (魏平), Kong GH (孔国辉), Ye WH (叶万辉) (1999). Production and turnover rate of fine roots in two lower subtropical forest sites at Dinghushan. Acta Phytoecologica Sinica (植物生态学报), 23, 361 - 369. (in Chinese with English abstract)

Zhang XQ (张小全) (2001). Fine root biomass, production and turnover of trees in relations to environmental conditions. Forest Research (林业科学研究), 14,566-573. (in Chinese with English abstract) 